\title{
Performance Analysis of Paraxial Free Space Optical Interconnects System that Uses Bessel Beams and Micro-Lenses with Circular Apertures
}

\author{
Nedal Al-Ababneh
}

\begin{abstract}
In this article the performance of microlens based free space optical interconnects system under paraxial approximations is considered. Instead of using Gaussian beams, we propose the use of the Bessel beams to transfer the information. The performance of the proposed system is evaluated and compared with that of Gaussian beams using the signal to crosstalk ratio as the system metric. It is shown that the optical system that uses Bessel beams gives superior results when used in applications with large interconnects length.
\end{abstract}

Index Terms-Bessel beams, diffraction, free space optical interconnects, gaussian beams, non-diffracting beams.

\section{INTRODUCTION}

Crosstalk due to light propagation diffraction and diffraction from the apertures of microlenses is considered as one of the important problems that affects the performance of free space optical interconnects (FSOIs) system, namely, the signal to crosstalk ratio [1]-[8]. In these arrayed optical systems the crosstalk noises from neighboring channels sum up in the victim channel and degrade the signal-to-noise ratio (SNR) at the receiver input. Typically, light beams with Gaussian profiles are used to carry the useful signal in FSOIs. However, the diffraction of the Gaussian beam increases with interconnect length and may result in a malfunction system beyond given interconnect length. One interesting concept to reduce the diffraction, particularly in applications with large propagation distance, is the use of non-diffracting beams. The concept of using non-diffracting beam in lensless free space optical interconnects was investigated in [5]. It was shown that the performance of the FSOIs system using non-diffracting beams outperforms the one that uses Gaussian beams especially for large interconnects distance.

In this paper, we investigate the potential of using non-diffracting Bessel beams in free space optical interconnects that uses micro-lenses with circular aperture. An analytical expression for the light intensity distribution at the detector plane is derived by expanding the microlens aperture in term complex Gaussian functions. Using this expression the signal to crosstalk ratio is evaluated and used as the performance measure. We also introduce results for the signal to crosstalk ratio for the system using the Gaussian beams. In Section II the optical field at the detector plane is

Manuscript received December 9, 2013; revised March 25, 2014.

N. Al-Ababneh is with Department of Electrical Engineering, Jordan University of Science \& Technology, Irbid, Jordan (e-mail: nedalk@just.edu.jo). derived. In Section III the signal to crosstalk calculations are introduced. Numerical simulations are presented in Section VI. Section V concludes the paper.

\section{OPTICAL FIELD AT DeTECTOR Plane}

Consider the FSOIs system shown in Fig. 1. This optical system consists of non-diffracting light sources array, microlens array, and detectors array.

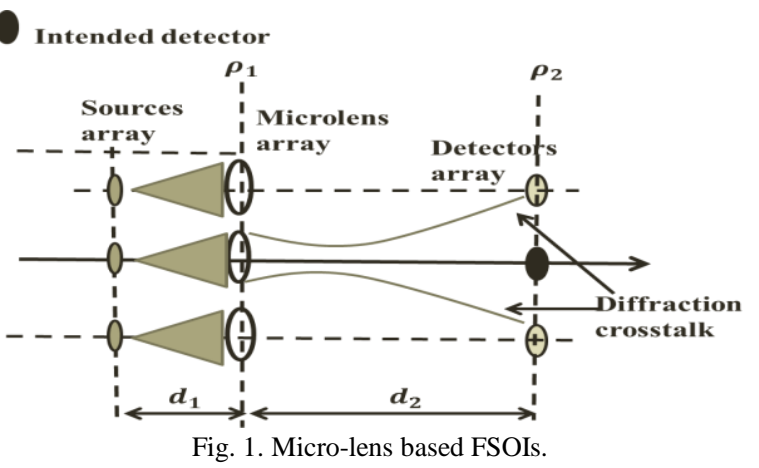

The light sources array is placed at a distance $d_{1}$ from the microlens array. The distance between the detector array and the microlenses array is $d_{2}$. To find the field distribution at the detectors plane we use the diffraction integral formula for an aligned optical system in cylindrical coordinate system assuming paraxial approximations. Based on this formula the output optical field at the detectors plane can be related to an input optical field by [6]

$$
\begin{aligned}
\mathrm{E}_{2}\left(\rho_{2}, \theta_{2}\right)= & \frac{i k}{2 \pi b} \int_{0}^{\infty} \int_{0}^{2 \pi} \mathrm{E}_{1}\left(\rho_{1}, \theta_{1}\right) A\left(\rho_{1}\right) \\
& \times \exp \left[\begin{array}{c}
-\frac{i k a}{2 b} \rho_{1}^{2}-\frac{i d k}{2 b} \rho_{2}^{2} \\
+\frac{i k \rho_{1} \rho_{2}}{b} \cos \left(\theta_{1}-\theta_{2}\right)
\end{array}\right] \rho_{1} d \rho_{1} d \theta_{1}
\end{aligned}
$$

$\rho_{1}, \theta_{1}$ and $\rho_{2}, \theta_{2}$ are the cylindrical coordinates at the input and the output planes, respectively. $A\left(\rho_{1}\right)$ is the aperture function of the micro-lens. $E_{1}\left(\rho_{1}, \theta_{1}\right)$ is the input optical field at the front surface of the microlens which resembles the output filed emitted by the source after propagating the $d_{1}$ distance. $k=2 \pi / \lambda$ is the wave number, and $\lambda$ is the wavelength of the emitted beam. $a, b, c$, and $d$ are the transfer matrix elements of the FSOIs system under consideration and are given by: 


$$
\left[\begin{array}{ll}
a & b \\
c & d
\end{array}\right]=\left[\begin{array}{ll}
1-\left(d_{2} / f\right) & d_{2} \\
-1 / f & 1
\end{array}\right]
$$

$f$ is the focal length of the microlens. Assuming a Bessel non-diffracting beam for the input optical filed, $\mathrm{E}_{1}\left(\rho_{1}, \theta_{1}\right)$ can be given by

$$
\mathrm{E}_{1}\left(\rho_{1}, \theta_{1}\right)=\mathrm{J}_{0}\left(\sigma \rho_{1}\right)
$$

$\mathrm{J}_{0}$ is the first kind zeroth order Bessel function. $\sigma$ is the transverse wave number which controls the sharpness of the beam. For a uniform hard edge circular aperture function with $h_{1}$ radius

$$
A\left(\rho_{1}\right)= \begin{cases}1 & \rho_{1} \leq h_{1} \\ 0 & \rho_{1} \succ h_{1}\end{cases}
$$

The integral in Eq. (1) can be solved by expanding the uniform circular aperture function of the microlens in terms of finite number of complex Gaussian functions [9], [10]. Let $C_{1 n}$ and $C_{2 n}$ be some complex coefficients, then $A P\left(\rho_{1}\right)$ can be expanded as

$$
A P\left(\rho_{1}\right)=\sum_{n=1}^{N} C_{1 n} \exp \left(-\frac{C_{2 n}}{h_{1}^{2}} \rho_{1}^{2}\right)
$$

Now, by substituting Eq. (5) into Eq. (1) and using the following two integrals

$$
\begin{aligned}
& \frac{1}{2 \pi} \int_{0}^{2 \pi} \exp \left[i x \cos \left(\theta_{2}-\theta_{1}\right)\right] \exp \left[-i n \theta_{1}\right] d \theta_{1} \\
&= i^{n} J_{n}(x) \exp \left[-i n \theta_{2}\right] \\
& \int_{0}^{\infty} \exp \left[-\gamma \rho^{2}\right] J_{n}(\alpha \rho) J_{n}(\beta \rho) \rho d \rho \\
&=\frac{(-1)^{n}}{2 \gamma} \times \exp \left[-\frac{i n \pi}{2}\right] \exp \left[-\frac{1}{4 \gamma}\left(\alpha^{2}+\beta^{2}\right)\right] J_{n}\left(\frac{i \alpha \beta}{2 \gamma}\right)
\end{aligned}
$$

The output optical field at the detectors array can be obtained as

$$
\begin{gathered}
\mathrm{E}_{2}\left(\rho_{2}, \theta_{2}\right)=\sum_{n=1}^{N} \frac{k(-1)^{n}(\mathrm{i})^{\mathrm{n}+1}}{b q} \exp \left[-i n \theta_{2}+\frac{i n \pi}{2}\right] \\
\exp \left[-\frac{i k}{b} d \rho_{2}^{2}\right] \exp \left[-\frac{1}{2 q}\left(\sigma^{2}+\frac{k^{2} \rho_{2}^{2}}{b^{2}}\right)\right] J_{n}\left(i \sigma \frac{k \rho_{2}}{q b}\right) \\
q=\frac{2 C_{2 n}}{h_{1}^{2}}+\frac{i k a}{b}
\end{gathered}
$$

The field intensity distribution is given by

$$
\mathrm{I}_{2}\left(\rho_{2}, \theta_{2}\right)=\left|\begin{array}{l}
\sum_{n=1}^{N} \frac{k(-1)^{n}(\mathrm{i})^{\mathrm{n}+1}}{b q} \\
\exp \left[-\frac{1}{2 q}\left(\sigma^{2}+\frac{k^{2} \rho_{2}^{2}}{b^{2}}\right)\right] J_{n}\left(i \sigma \frac{k \rho_{2}}{q b}\right)
\end{array}\right|
$$

\section{Signal to CRosstalk Ratio CAlculations}

The crosstalk can be seen as the light coupled into the intended detector from all light sources excluding the intended source. Equivalently, the crosstalk power received by the intended detector can be seen as the power received by all other detectors from the light passing through intended microlens with only the intended source is on. Therefore, the crosstalk power can be evaluated by

$$
\begin{aligned}
P_{c}= & 4 \iint_{A_{1}} \mathrm{I}_{2}\left(\rho_{2}, \theta_{2}\right) \rho_{2} d \rho_{2} d \theta_{2} \\
& +4 \iint_{A_{2}} \mathrm{I}_{2}\left(\rho_{2}, \theta_{2}\right) \rho_{2} d \rho_{2} d \theta_{2}
\end{aligned}
$$

$A_{1}$ is the area covered by one of the four neighbor detectors and $A_{2}$ is the area covered by one of the next four neighbor detectors. The signal power is the power of the intended detector from the light coming from the intended source through the intended microlens and is given by:

$$
P_{s}=\iint_{A} \mathrm{I}_{2}\left(\rho_{2}, \theta_{2}\right) \rho_{2} d \rho_{2} d \theta_{2}
$$

$A$ is the area covered by intended detector. Having evaluated the signal and the crosstalk power, the signal to crosstalk ratio, SCR, can be determined as

$$
S C R=\frac{P_{s}}{P_{c}}
$$

\section{NUMERICAL SIMULATIONS}

In this section we introduce numerical examples to show the benefits of using the Bessel beams in microlens based free space optical interconnects system. We also introduce results for the optical system using Gaussian beams. In the simulations, the Bessel beam has a wavelength $\lambda=0.85 \mu \mathrm{m}$ and a transverse wave number $\sigma=2.5 \mu \mathrm{m}^{-1}$ and the Gaussian beam has the same wavelength with a beam radius of $5 \mu \mathrm{m}$ at the front surface of the microlens. Moreover, the microlens has a focal length and a diameter of $720 \mu \mathrm{m}$ and $300 \mu \mathrm{m}$, respectively. Ten terms were used to approximate the aperture function. The complex coefficients for these terms are given in [7]. The distance between neighboring detectors as well as neighboring light sources is $300 \mu \mathrm{m}$ which corresponds to an interconnect density of $1111 \mathrm{~cm}^{-2}$. Fig. 2 shows the profiles for the input optical field intensity for the Bessel and Gaussian beams.

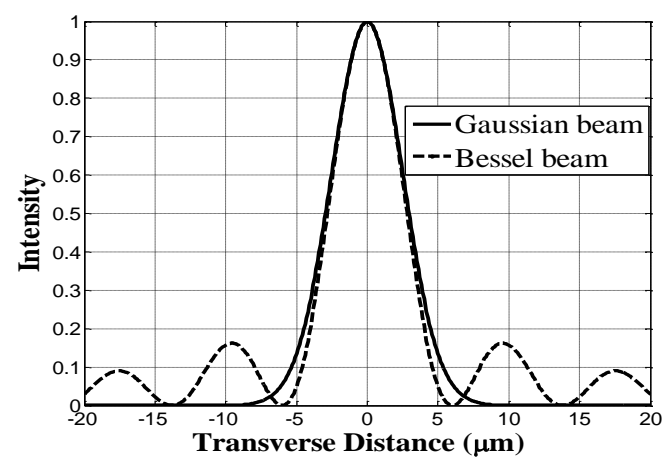

Fig. 2. Input optical field intensity for the two beams. 
To show the importance of using Bessel beams, the SCR versus the detector radius is plotted in Fig. 3-Fig. 5 for three different values of interconnects length. Comparing the three figures, one can see that the SCR for the FSOIs system using Bessel beam outperforms that of the system which uses the Gaussian beam for large interconnects length. In Fig. 3, the interconnect length is small and using the Gaussian beam is still preferred since the diffraction of the light can be tolerated.

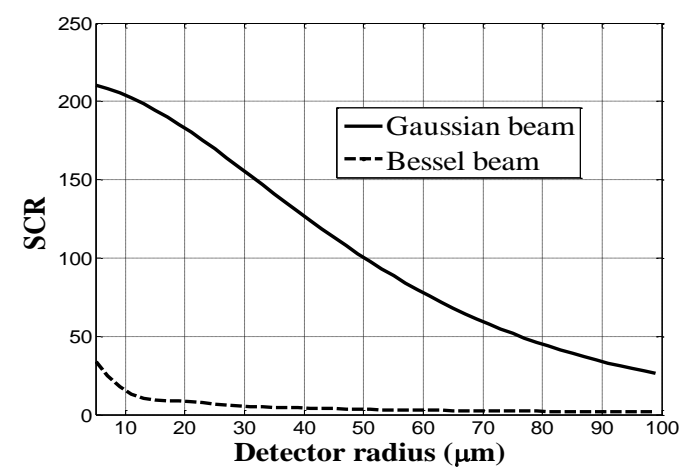

Fig. 3. SCR versus detector radius with interconnect length of $3 \mathrm{~mm}$.

However, as the interconnects length increases the dramatic effect of the diffraction is apparent as shown in Fig. 4 and Fig. 5 and in this case the Bessel beam is preferred.

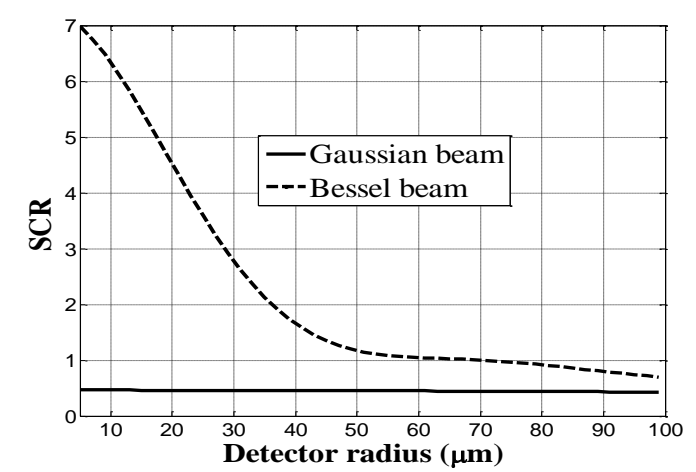

Fig. 4. SCR versus detector radius with interconnect length of $8 \mathrm{~mm}$.

To more explain the results obtained in the above figures, we plotted in Fig. 6 and Fig. 7 the normalized ( with respect to that of the shortest interconnect length) optical filed distribution at the detector plane for different interconnect lengths. It is clear from Fig. 6 that the power that may capture by the intended detector is comparable to that may receive by the any neighbor detector. For the case of arrayed optical system which consist of thousands of channels, the light sum up in the victim detector and result in a malfunction system as shown in Fig. 8.

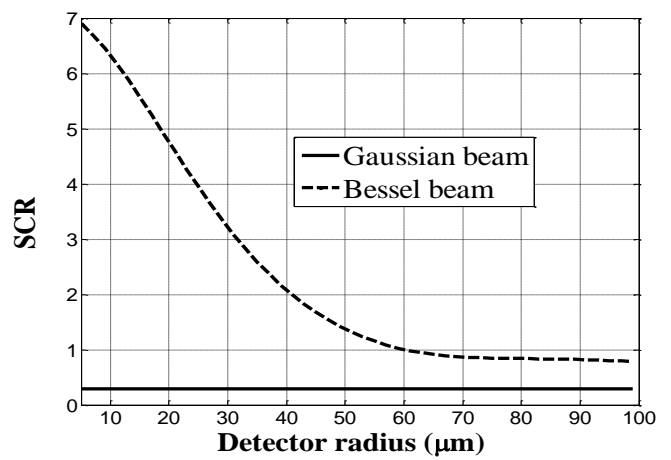

Fig. 5. SCR versus detector radius with interconnect length of $10 \mathrm{~mm}$.

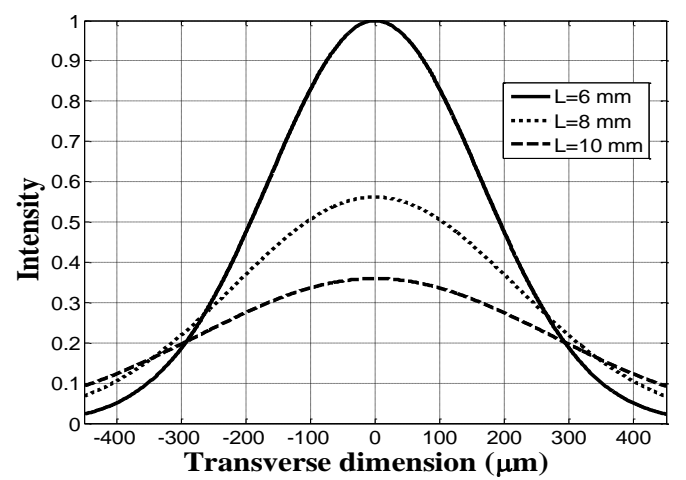

Fig. 6. Normalized output field intensity for Gaussian beam.

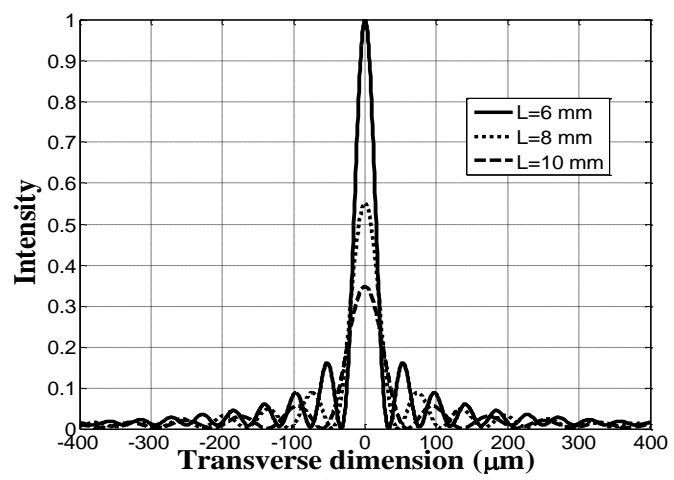

Fig. 7. Normalized output field intensity for Bessel beam.

For a threshold SCR of 5, the optical system with interconnect length of $5 \mathrm{~mm}$ and more will not operate with an acceptable bit error rate.

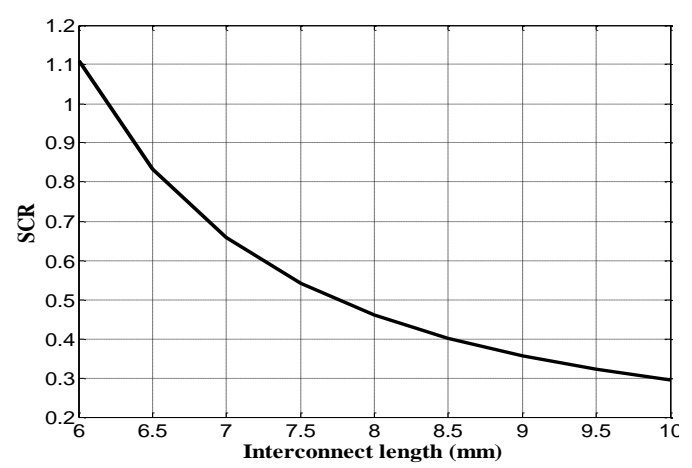

Fig. 8. SCR versus interconnect length for the Gaussian beam.

\section{CONCluSION}

The performance of a microlens based free space optical interconnects system which uses Bessel beams to carry the information signal was analyzed using paraxial approximation. An analytical formula for optical field at the detector plane was derived and used to evaluate the signal to crosstalk ratio which we used as the performance measure. The simulation results have shown that the use of Bessel beam gave superior results to that of using Gaussian beam at large interconnects lengths. It is shown that the use of Gaussian beams in applications with large interconnect length may result in a malfunction system.

\section{REFERENCES}

[1] W. H. Hu, X. J. Li, J. K. Yang, and D. Kong, "Crosstalk analysis of aligned and misaligned free-space optical interconnect systems," $J$. Opt. Soc. Am. A, vol. 27, no. 2, pp. 200-205, 2010. 
[2] N. S. Petrovic and A. D. Rakic, "Modeling diffraction and imaging of laser beams by the mode-expansion method," J. Opt. Soc. Am. B, vol. 22, no. 3, pp. 556-566, 2005.

[3] F. F. Tsai, C. J. O'Brien, N. S. Petrovic, and A. D. Rakic, “Analysis of optical channel cross talk for free-space optical interconnects in the presence of higher-order transverse modes," Appl. Opt., vol. 44, no. 30, pp. 6380-6387, 2005.

[4] S. Tang et al., "Design limitations of highly parallel free-space optical interconnects based on array of vertical cavity surface-emitting laser diodes, microlenses, and photo detectors," J. Lightwave Technol., vol. 12, no. 11 , pp. 1971-1975, 1994.

[5] N. Al-Ababneh and M. Testorf, "Analysis of free space optical interconnects based on non- diffracting beams," in Proc. Optics Communications 242, 2004, pp. 393-400.

[6] N. Al-Ababneh, "Crosstalk reduction in free space optical interconnects systems using microlenses with Gaussian transmittance," Optics Communications 316, pp. 79-82, 2014.

[7] N. Al-Ababneh, "A simple approximate expression for optical crosstalk in a linear free space optical interconnects that uses micro-lenses with circular apertures," presented at International conference on machine learning, electrical and mechanical engineering, ICMLEME'2014, Dubai, UAE, Jan. 8-9, 2014.
[8] S. A. Collins, "Lens-systems diffraction integral written in terms of matrix optics," J. Opt. Soc. Am., vol. 60, no. 9, pp. 1168-1177, 1970.

[9] J. J. Wen and M. A. Breazeale, "A diffraction beam field expressed as the superposition of Gaussian beams," J. Acoust. Soc. Am., vol. 83, no. 5, pp. 1752-1756, 1988.

[10] D. Ding and Y. Zhang, "Notes on the Gaussian beam expansion," $J$. Acoust. Soc. Am., vol. 116, no. 3, pp. 1401-1405, 2004.

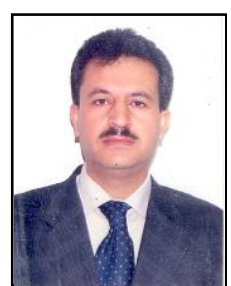

Nedal Al-Ababneh was born in the city of Irbid, Jordan in June 1970. He received his B.Sc. and M.Sc. degrees in electrical engineering from Jordan University of Science and Technology in 1993 and 1996 respectively. He received his doctoral of engineering degree in electrical engineering from University of Massachusetts-Lowell (USA) in 2004 $\mathrm{He}$ is currently an associate professor with the Department of Electrical Engineering in Jordan University of Science and Technology. His research interests are in free space optical interconnects and distributed detection systems. 

Modeling and Simulation 
